\title{
MEG final results and progress towards MEG II
}

\author{
T. Iwamoto ${ }^{1^{*}}$ on behalf of the MEG II collaboration \\ 1 ICEPP, The University of Tokyo, 7-3-1 Hongo Bunkyo-ku, Tokyo, 113-0033, Japan * \\ E-mail: iwamoto@icepp.s.u-tokyo.ac.jp
}

November 29, 2018

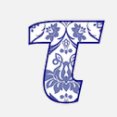

Proceedings for the 15th International Workshop on Tau Lepton Physics, Amsterdam, The Netherlands, 24-28 September 2018

scipost.org/SciPostPhysProc. Tau2018

\begin{abstract}
The MEG experiment, which is to search for lepton flavor violating muon decay, had been successfully finished in 2013. The final sensitivity of the experiment was $5.3 \times 10^{-13}$, and since the experiment did not find any signal, the upper limit of the branching ratio of the $\mu^{+} \rightarrow e^{+} \gamma$ was set to be $4.2 \times 10^{-13}$ at $90 \% \mathrm{CL}$. The MEG II experiment will improve the sensitivity by an order of magnitude with three years data taking, and the target sensitivity is $6 \times 10^{-14}$. In 2018 after the detector integration, the muon beam data taking will be planned with limited number of readout channels. In 2019, the engineering run and the physics run will be started.
\end{abstract}

\section{Introduction}

The standard model (SM) of the elementary particle physics can explain the most experimental results, and there is no strong evidence that indicates the new physics beyond SM up to now. However, the SM can not explain why we have three generations, the existence of dark matter, many parameters contained in the model itself, gravity, and we consider that the model is a low energy appproximation of a more fundamental theory. Many physicists in the world are looking for such new physics beyond the SM.

One method is to use the highest energy accelerator like the Large Hadron Collider to produce new particles which belong to the new physics. The other method is to use a high intensity beam to detect rare phenomena which can happen in new physics. One example is to look for lepton flavor violation (LFV) phenomena. LFV is practically forbidden in the $\mathrm{SM}$, but in the neutrino sector, it is already seen in the neutrino oscillation phenomena. In the charged lepton sector, it has never been detected although there is no theoretical reason why it is forbidden in the SM. On the other hand, many new physics scenarios like SUSY-GUT or SUSY-seesaw predict large branching ratio of $\mu^{+} \rightarrow e^{+} \gamma$.

The MEG experiment was designed to search for such regions where new physics like SUSY-GUT, SUSY-seesaw predict through $\mu^{+} \rightarrow e^{+} \gamma$, and it had a real chance to discover new physics. The data taking had been performed between 2009 and 2013, and the final sensitivity was $5.3 \times 10^{-13}$. Unfortunately the MEG experiment did not find any signal, and the upper limit of the branching ratio of the $\mu^{+} \rightarrow e^{+} \gamma$ is set to be $4.2 \times 10^{-13}$ [1].

The MEG II experiment will improve the sensitivity by an order of magnitude with three years data taking, and the target sensitivity is $6 \times 10^{-14}[2]$. 


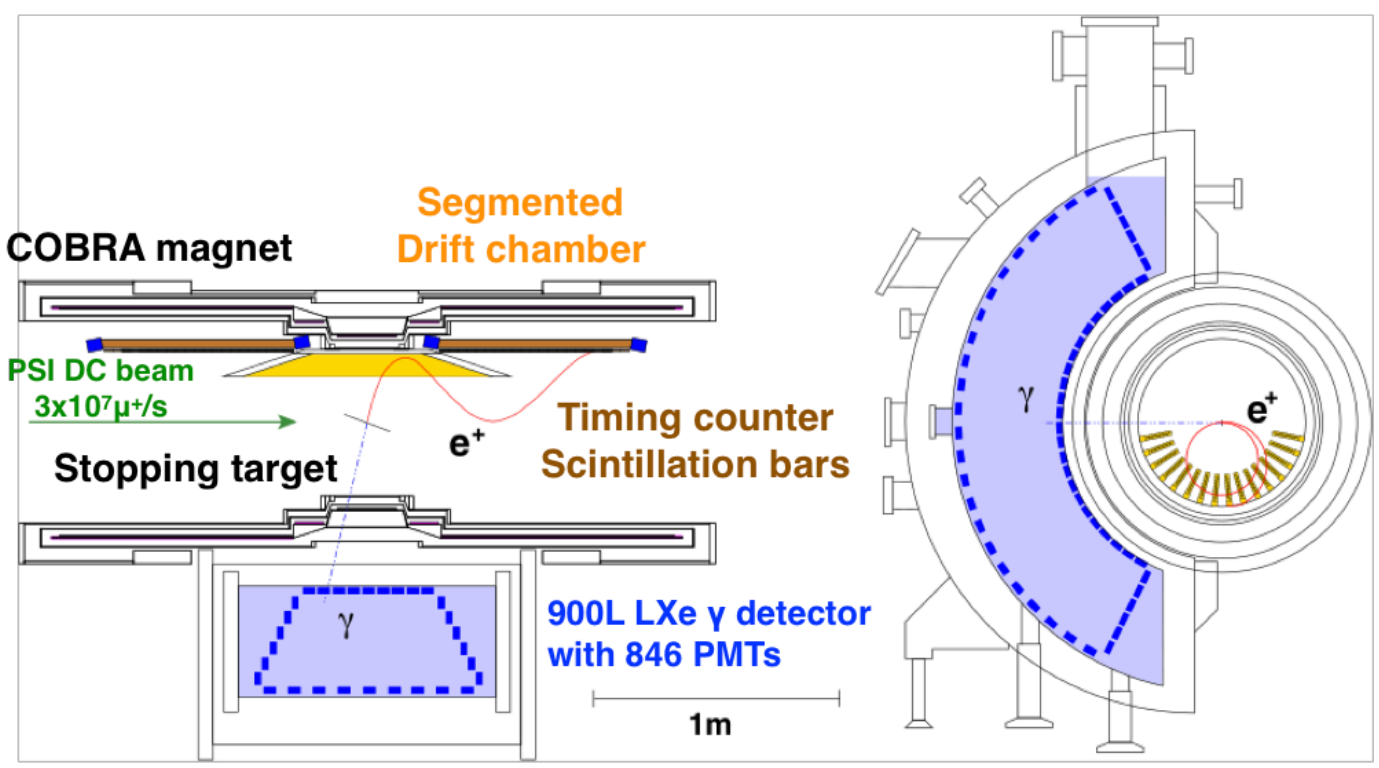

Figure 1: The schematic view of the MEG detector

\section{MEG Experiment}

The MEG experiment was performed at the Paul Scherrer Institute (PSI) in Switzerland which has a world most intense $590 \mathrm{MeV}$ proton accelerator with a current of $2.2 \mathrm{~mA}$. The accelerator can produce DC muon beam up to $1 \times 10^{8} / \mathrm{s}$, but the MEG experiment used $3 \times 10^{7} / \mathrm{s}$ to reduce the accidental background, and thus to maximize the experimental sensitivity. Positive Muons are stopped at the thin target located in the center of the magnetic spectrometer. We will measure a positron and a $\gamma$ emitted from it at the same time, back-to-back, with the energy of $52.83 \mathrm{MeV}$ by the MEG detector(Fig. 1). Positrons are bent by the superconducting magnet, and the trajectory is tracked by the segmented drift chambers. Positron times are measured by a timing counter which consists of scintillation bars read out by PMTs. $\gamma$ s are measured with 900L liquid xenon $\gamma$ detector with 846 PMTs. There are mainly two background to search for $\mu^{+} \rightarrow e^{+} \gamma$ decays. One is an accidental background of a usual Michel decay $\mathrm{e}^{+}$and a random $\gamma$ from radiative muon decay or annihilation in flight. The other is a radiative muon decay background, and a $\gamma$ is emitted in addition to the normal Michel decay. The time of the $\mathrm{e}^{+}$and $\gamma$ is coincident. Our dominant background is the accidental background. The total number of accidental background $\left(N_{a c c}\right)$ is proportional to

$$
N_{a c c} \propto R_{\mu}^{2} \times \Delta E_{\gamma}^{2} \times \Delta E_{e} \times \Delta \Theta_{e \gamma}^{2} \times \Delta t_{e \gamma} \times T
$$

where $R_{\mu}$ is a muon rate, $\Delta E_{\gamma}$ is a resolution of $\gamma$ energy, $\Delta E_{e}$ is a resolution of $\mathrm{e}^{+}$energy, $\Delta \Theta_{e \gamma}$ is a resolution of opening angle between $\mathrm{e}^{+}$and $\gamma, \Delta t_{e \gamma}$ is a timing resolution between $\mathrm{e}^{+}$and $\gamma$, and $\mathrm{T}$ is total measurement time. The number of the accidental background is proportional to the square of the muon beam intensity, and the lower instantaneous muon beam rate is better to suppress the accidental background. The DC muon beam such as PSI proton accelerator is suitable for this purpose. Better detector resolutions are also important to reduce the accidental background.

The accumulated number of muons stopped on the target between 2009 and 2013 is $7.5 \times 10^{14} \mu^{+}$, which is the full dataset for the MEG experiment. Five observables of $\mathrm{E}_{\gamma}$, $\mathrm{E}_{e}, \mathrm{t}_{e \gamma}, \theta_{e \gamma}$, and $\phi_{e \gamma}$ are used for the analysis, and the blind analysis is adopted in $\mathrm{E}_{\gamma}$, 

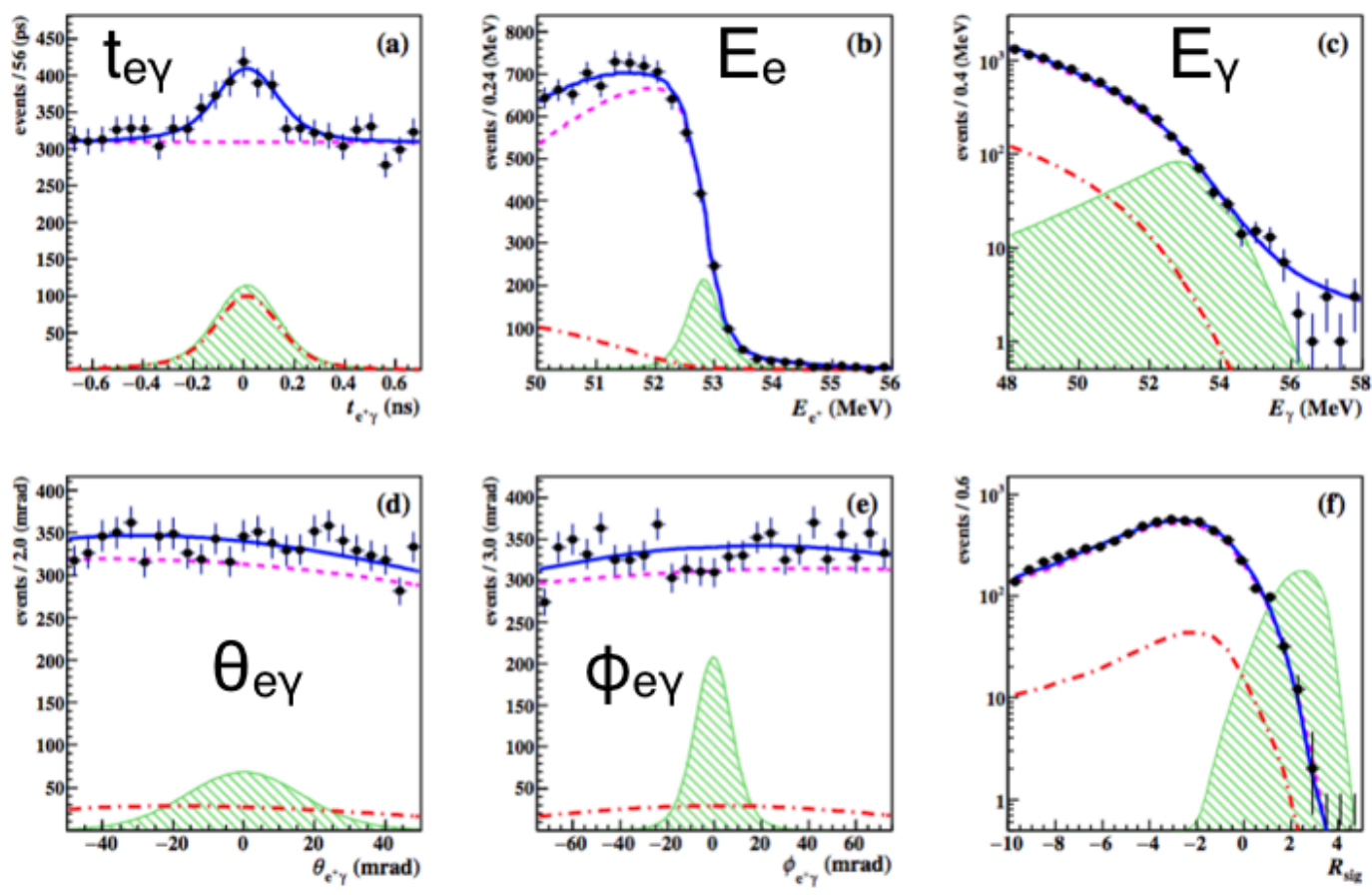

Figure 2: Each variable projected from the likelihood results for the full dataset

$\left.\mathrm{t}_{e} \gamma\right)$ plane. After opening the blinded region, the maximum likelihood analysis is applied for those events within the analysis window.

Fig. 2 shows the likelihood results projected into each variable. The blue solid line is the total fit result, the magenta dash line is the number of accidental backgrounds, and the red dot-dash line is the number of RMD. From the likelihood fit, the numbers of signal, RMD, and accidental background are extracted. The green hatched histograms show the signal PDFs magnified with 100 times of $\mathrm{N}_{\text {sig }}$ upper limit. All PDFs are well consistent with the data, and the result was consistent with no signal. The best fit values for $\mathrm{N}_{A C C}$ and $\mathrm{N}_{R M D}$ were $7684 \pm 103,663 \pm 59$, respectively. The upper limit of the branching ratio of the $\mu^{+} \rightarrow e^{+} \gamma$ is set to be $4.2 \times 10^{-13}$ at $90 \%$ C.L.

\section{$3 \quad$ MEG II Experiment}

The improvement of the sensitivity in the MEG experiment was already limited by the accidental background. In order to improve the MEG sensitivity further, we need to perform the detector upgrade to reduce the dominant accidental background. If the detector performance is improved sufficiently, we can make use of the maximum beam intensity at PSI. To reduce the multiple scattering and the $\gamma$ background produced in the target, thinner target will be used in the MEG II. The stopping rate will be $7 \times 10^{7} / \mathrm{s}$, which is more than twice intensity of the MEG. The drawing of the MEG II detector is described in Fig. 3.

The positron spectrometer will use the superconducting magnet again which has a special gradient magnetic field. In order to track $52.8 \mathrm{MeV}$ positron and to reconstruct vertex, angle, and momentum, a new single volume cylindrical drift chamber $(\mathrm{CDCH})$ is built for the MEG II positron tracker which has smaller stereo cells, and thus has more hits resulting in better momentum, angular resolutions. The total length of the drift chamber 


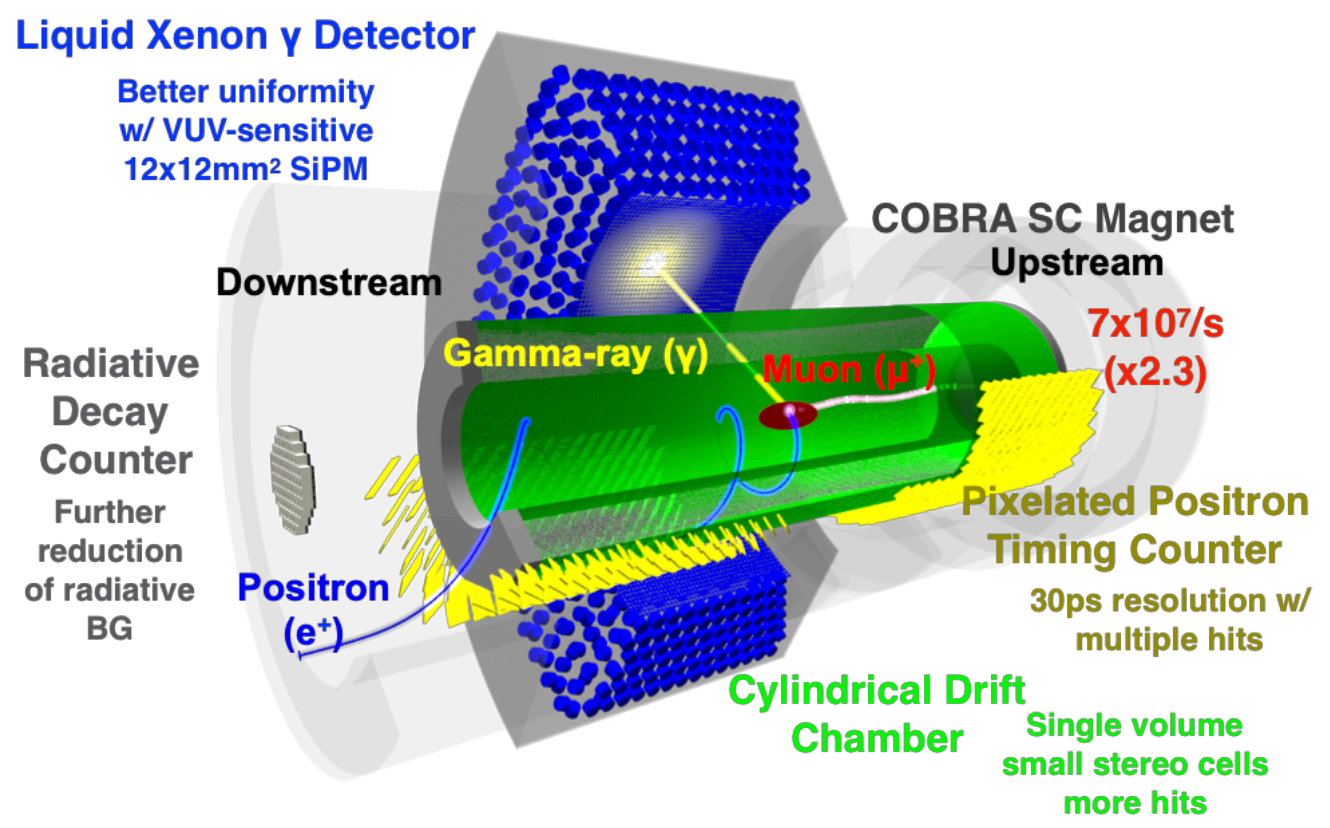

Figure 3: Drawing of the MEG II detector

is $2 \mathrm{~m}$, and the chamber gas will be $\mathrm{He}_{\mathrm{iC}} \mathrm{H}_{10}=85: 15$. A new pixelated positron timing counter (TC) is prepared to detect $52.8 \mathrm{MeV}$ positron time precisely with multiple hits down to 30 ps. Segmented 256 scintillator plates with a dimension of $12 \times(4$ or 5$) \times 0.5 \mathrm{~cm}^{3}$ readout by SiPM are installed into both upstream and downstream side. The higher positron detection efficiency is realized by less drift chamber material, high transparency towards timing counter.

The inner face of the liquid xenon $\gamma$ detector (LXe), which was covered by 2162 inch PMTs, is now replaced with $409212 \times 12 \mathrm{~mm}^{2}$ VUV-sensitive MPPCs newly developed in cooporation with Hamamatsu [3]. This enables better granularity and uniformity of the detected photoelectrons independent of the $\gamma$ incident positions, and better energy, position resolutions will be achieved.

High energy $\gamma$ s from radiative muon decays can be the dominant accidental background source for $\gamma$ side because the material inside the drift chamber is reduced, and annihilation in flight can be reduced which is another dominant $\gamma$ background. A tagging detector of the RMD to detect low momentum positron with high energy $\gamma$ detection in the LXe detector can reduce the RMD background events. The new device for this, called Radiative Decay Counter (RDC), is newly introduced at the downstream side in the MEG II detector. Plastic scintillators readout by SiPM are mainly to detect positron timing, and LYSO crystals with SiPMs are to measure positron energy.

Many detectors in the MEG II experiment have finer granularity with SiPMs and more readout channels are required. Experiments like MEG II under high rate environment require waveform digitizers to reduce pileup events. To fulfill these requirements, compact electronics with more functionality including waveform digitizer, simple trigger, bias voltage supply for SiPM, amplifier, called WaveDREAM (Waveform digitizer with DRS4 readout module) are developed at PSI. The online trigger is important to manage high event rate and background suppression, and the FPGA based trigger system is prepared for the MEG II experiment. 


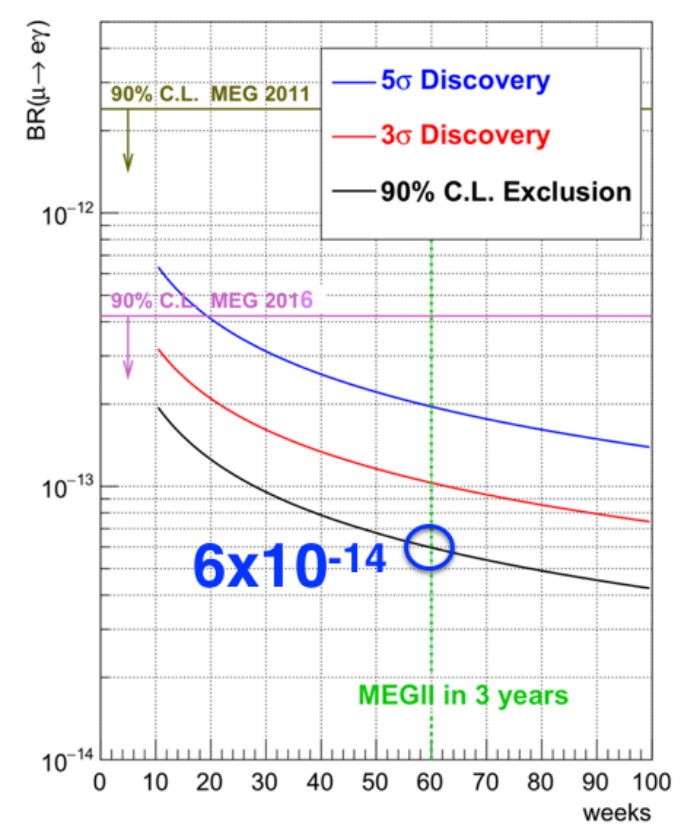

Figure 4: Sensitivity plot as a function of the DAQ time

Fig. 4 shows the MEG II sensitivity as a function of the DAQ time.

Conservatively 20 DAQ weeks are assumed for a year. In a few weeks DAQ, the current upper limit will be exceeded by the MEG II experiment, and the sensitivity will reach $6 \times 10^{-14}$ in three years.

\section{Current Status}

CDCH construction is finished at PISA in July 2018, and sent to PSI. The HV conditioning was performed once outside the experimental site, and currently the $\mathrm{CDCH}$ detector is installed inside the superconducting magnet. TC construction has been fisniehd in 2017, and basic performance test was already done with muon beam [4]. LXe detector construction has also been finished in 2017. Detector commissioning is in progress [5]. RDC downstream detector is constructed in 2017, and the performance test with muon beam was finished in 2017 [6]. The electronics development is in the final stage. Last year, 96 prototype WaveDREAM boards (1536 channels) were produced, and used for the beam time in 2017. This year, all the detectors are integrated in the experimental hall, and the prototype boards are used for the beam time, too. Based on the final test this year, the mass production will be started next year [7].

\section{Conclusion}

The MEG experiment finished physics data taking in 2013, and the final result was published in 2016, which set the most stringent upper limit of the $\mu^{+} \rightarrow e^{+} \gamma$ branching ratio, $4.2 \times 10^{-13}$ at $90 \%$ C.L. In order to improve the sensitivity by an order of magnitude, the MEG II experiment, which is to upgrade all the detectors of the MEG experiment, is proposed, and is accepted at PSI. The MEG II detector integration is now in progress. The pilot run in 2018 will have all the detectors with limited number of electronics channels, 
and each detector performance will be evacuated carefully. The muon beam time will be started soon in December 2018. The mass production of the readout electronics will be finished next year, and the engineering run will be started followed by physics run. The sensitivity of the MEG II experiment will exceed the current limit with a few month data, and will be improved by one order of magnitude with three years data.

\section{Acknowledgements}

This work is supported by MEXT/JSPS KAKENHI Grant Numbers JP22000004, JP26000004, and JSPS Core-to-Core Program, A. Advanced Research Networks.

\section{References}

[1] A. M. Baldini et al., Search for the lepton flavour violating decay $\mu^{+} \rightarrow \mathrm{e}^{+} \gamma$ with the full dataset of the MEG experiment, Eur. Phys. J. C76(8), 434 (2016), doi:10.1140/epjc/s10052-016-4271-x, 1605.05081.

[2] A. M. Baldini et al., The design of the MEG II experiment, Eur. Phys. J. C78(5), 380 (2018), doi:10.1140/epjc/s10052-018-5845-6, 1801.04688.

[3] W. Ootani et al., Development of deep-UV sensitive MPPC for liquid xenon scintillation detector, Nucl. Instrum. Meth. A787, 220 (2015), doi:10.1016/j.nima.2014.12.007.

[4] M. Nakao et al., Results from Pilot Run for MEG II Positron Timing Counter, Springer Proc. Phys. 213, 237 (2018), doi:10.1007/978-981-13-1316-5_44, 1808.07279.

[5] S. Ogawa, Liquid Xenon Detector with VUV-Sensitive MPPCs for MEG II Experiment, Springer Proc. Phys. 213, 76 (2018), doi:10.1007/978-981-13-1316-5_14.

[6] R. Iwai, K. Ieki and R. Sawada, Radiative Decay Counter for Active Background Identification in MEG II Experiment, Springer Proc. Phys. 212, 82 (2018), doi:10.1007/978-981-13-1313-4_17.

[7] L. Galli et al., WaveDAQ: An highly integrated trigger and data acquisistion system, Nucl. Instrum. Meth., In Press (2018), doi:10.1016/j.nima.2018.07.067. 